\title{
Impact of Permissive Hypoxia and Hyperoxia Avoidance on Clinical Outcomes in Septic Patients Receiving Mechanical Ventilation: A Retrospective Single-Center Study
}

Kota Nishimoto

Kansai Medical University

Takeshi Umegaki ( $\square$ umegakit@hirakata.kmu.ac.jp )

Kansai Medical University

Sayaka Ohira

Kansai Medical University

Takehiro Soeda

Kansai Medical University

Natsuki Anada

Kansai Medical University

Takeo Uba

Kansai Medical University

Tomohiro Shoji

Kansai Medical University

Munenori Kusunoki

Kansai Medical University

Yasufumi Nakajima

Kansai Medical University

Takahiko Kamibayashi

Kansai Medical University

\section{Research Article}

Keywords: septic patients, hyperoxia, ventilatory strategies, ventilator access

Posted Date: January 18th, 2021

DOl: https://doi.org/10.21203/rs.3.rs-146649/v1

License: (c) (1) This work is licensed under a Creative Commons Attribution 4.0 International License.

Read Full License 
Page $2 / 26$ 


\section{Abstract}

Different ventilatory strategies may affect survival in septic patients requiring mechanical ventilation. In this retrospective before-and-after study, we examined the effects of permissive hypoxia and hyperoxia avoidance on mortality in mechanically ventilated adult ( $\geq 18$ years) septic patients at a Japanese university hospital. In April 2017, our hospital's mechanical ventilation policy changed from a conventional oxygenation target $\left(\mathrm{SpO}_{2}: \geq 96 \%\right.$.) to more conservative targets with permissive hypoxia $\left(\mathrm{SpO}_{2}: 88-92 \%\right.$ or $\mathrm{PaO}_{2}: 60 \mathrm{mmHg}$ ) and hyperoxia avoidance (reduced oxygenation for $\mathrm{PaO}_{2}>110$ $\mathrm{mmHg}$ ). Patients were divided into a pre-change group (April 2015 to March 2017; n=83) or post-change group (April 2017 to March 2019; n=130). Using a multiple logistic regression model, we examined the association of the post-change group with intensive care unit (ICU) mortality. The post-change group did not have a significantly lower adjusted ICU mortality (odds ratio: $0.67,95 \%$ confidence interval: 0.33-1.43; $P=0.31$ ) than the pre-change group. However, there were significant differences in mechanical ventilation duration (pre-change: 11.0 days, post-change: 7.0 days; $P=0.01$ ) and ICU stay (pre-change: 11.0 days, post-change: 9.0 days; $P=0.02$ ). Although the new ventilation policy did not affect ICU mortality, the reductions in mechanical ventilation duration and ICU stay may improve patient turnover and ventilator access.

\section{Introduction}

Patients with sepsis often require mechanical ventilation for respiratory dysfunction, and the use of different ventilatory strategies can affect survival rates ${ }^{1}$. As mortality rates have been reported to exceed $30 \%$ in mechanically ventilated septic patients ${ }^{2,3}$, the improvement of mechanical ventilation protocols may have important implications for survivability.

A randomized controlled trial is currently examining the effects of permissive hypoxia in mechanically ventilated patients with respiratory dysfunction ${ }^{4}$, and a recent review indicated that permissive hypoxia may provide a better risk-benefit profile than the restoration of normoxia ${ }^{5}$. The dissociation curve of arterial oxygen tension $\left(\mathrm{PaO}_{2}\right)$ and oxyhemoglobin saturation $\left(\mathrm{SpO}_{2}\right)$ has been shown to be relatively flat when the latter exceeds $90 \%^{6}$, which supports the rationale of using permissive hypoxia. On the other hand, hyperoxia may have detrimental effects on survival among patients in critical condition ${ }^{7,8}$. Therefore, a combined oxygenation strategy involving permissive hypoxemia and hyperoxia avoidance may improve the prognosis of mechanically ventilated patients with sepsis. However, there is a lack of evidence on the clinical effects of this strategy in a real-world setting.

Until March 31, 2017, our hospital's ventilation policy was to attain an $\mathrm{SpO}_{2}$ target of $\geq 96 \%$. On April 1 , 2017 , this policy changed to an $\mathrm{SpO}_{2}$ target of $88-92 \%$ or a $\mathrm{PaO}_{2}$ target of $60 \mathrm{mmHg}$; in cases where $\mathrm{PaO}_{2}$ exceeds $110 \mathrm{mmHg}$, the fraction of inspiratory oxygen $\left(\mathrm{FiO}_{2}\right)$ or peak inspiratory pressure (PIP) settings are reduced. In this study, we hypothesized that this policy shift to permissive hypoxia and hyperoxia avoidance would improve clinical outcomes in adult septic patients requiring mechanical 
ventilation. To test this hypothesis, we conducted a retrospective study to compare mortality in patients before and after this policy change.

\section{Methods}

\section{Study design and data source}

We conducted a retrospective single-center analysis of mechanically ventilated septic patients who had been admitted to the general intensive care unit (ICU) of a university hospital in Japan between April 1, 2015 and March 31, 2019. All data were extracted from the medical records and administrative claims of Kansai Medical University Hospital, Japan. This study was conducted in accordance with the principles of the Declaration of Helsinki, and was approved by the institutional review board of Kansai Medical University Hospital (Approval Number: 2019030). As the database comprised retrospective claims information, the ethics committee waived the need for informed consent. The data were not anonymized before being received by the authors, and were accessed between April 2019 and November 2020.

\section{Patient selection}

We first identified hospitalized patients aged 18 years or older who had been diagnosed with sepsis and had undergone mechanical ventilation during the study period. In accordance with Sepsis-3 criteria ${ }^{9}$, sepsis was defined as a life-threatening organ dysfunction caused by a dysregulated host response to infection leading to an increased Sequential Organ Failure Assessment (SOFA) score of 2 points or more. As our hospital's mechanical ventilation policy changed on April 1,2017, the patients were divided into 2 groups: the pre-change group comprised patients who were admitted to the ICU between April 1, 2015 and March 31, 2017, and the post-change group comprised patients who were admitted to the ICU between April 1, 2017 and March 31, 2019. The pre-change group received mechanical ventilation with conventional oxygenation targets, whereas the post-change group received mechanical ventilation with permissive hypoxia and hyperoxia avoidance.

\section{Patient characteristics}

We collected information on the following baseline characteristics: age, sex, height, body weight, source of sepsis (lung, abdomen, urinary tract, soft tissue, mediastinum, endocardium, and others), acute respiratory distress syndrome (ARDS) severity based on the Berlin definition ${ }^{10}$, and SOFA score. Body mass index was calculated using the formula: body weight $(\mathrm{kg}) \times$ height $^{-2}(\mathrm{~m})$.

All patients in both groups were initially ventilated with airway pressure release ventilation (APRV). The initial mechanical ventilation settings were $\mathrm{FiO}_{2}$ of 0.40 , PIP of $20-25 \mathrm{cmH}_{2} \mathrm{O}$, low expiratory pressure of $0 \mathrm{cmH}_{2} \mathrm{O}$, low expiratory pressure duration of 0.5 seconds, and respiratory rate of $10-12$ breaths/minute. In the pre-change group, the mechanical ventilation settings were adjusted at the discretion of the attending physician. In contrast, the settings in the post-change group were determined based on the new mechanical ventilation policy. 
Data were also obtained on PIP and plateau pressure just after tracheal intubation, the maximum PIP within 48 hours of tracheal intubation, and the plateau pressure at the maximum PIP within 48 hours of tracheal intubation. We also collected data on $\mathrm{PaO}_{2}$ divided by $\mathrm{FiO}_{2}$ (P/F ratio) just before and after tracheal intubation and at the maximum PIP within 48 hours of tracheal intubation.

\section{Outcome measures}

The primary outcome measure was ICU mortality. The secondary outcome measures were in-hospital mortality, duration of mechanical ventilation, incidence of barotrauma, duration of ICU stay, and duration of hospital stay. Barotrauma included pneumothorax, subcutaneous emphysema, and mediastinal emphysema.

\section{Statistical analysis}

Continuous variables were calculated as means and standard deviations, and categorical variables were calculated as percentages or scores with interquartile ranges (IQRs). To compare the differences between the pre-change and post-change groups, we used Student's $t$-test or Welch's $t$-test with Levene's test for continuous variables and the chi-squared test or Fisher's exact test for categorical variables. The various SOFA scores (respiratory, cardiovascular, liver, renal, coagulation, and neurologic) were calculated as median values accompanied by the interquartile range, and were analyzed using the Mann-Whitney $U$ test. The Shapiro-Wilk test was used to check for normality in the outcome measures with continuous variables; based on the results of this test, we used Student's $t$-test, Welch's $t$-test, or the Mann-Whitney $U$ test, as appropriate.

Multivariate logistic regression analyses were conducted to examine the association between the mechanical ventilation policy change and ICU mortality. First, we constructed a logistic regression model with ICU mortality as the dependent variable and the post-change group (ref: pre-change group) as the main independent variable of interest; the covariates included patient characteristics and $P / F$ ratios just before tracheal intubation. Next, we conducted propensity score matching (propensity to be assigned to the post-change group) based on SOFA scores, and reperformed the regression analysis using the propensity score-matched patients; the covariates included patient characteristics and P/F ratios just before tracheal intubation. Patients were matched using 1:1 nearest neighbor matching within a caliper width of 0.25 standard deviations of the propensity score logit. The regression analysis was also performed using ARDS patients only. The odds ratios (ORs) and 95\% confidence intervals (Cls) for the independent variables were calculated.

Kaplan-Meier survival curves were plotted to examine the inter-group differences in survival and time to weaning from mechanical ventilation. We also plotted survival curves for time to weaning from mechanical ventilation according to 3 categories of $P / F$ ratios $(\leq 100,100.1-200$, and $>200)$ just before tracheal intubation.

Two-tailed $P$ values lower than 0.05 were regarded as statistically significant. All analyses were performed using SPSS Version 26.0 (IBM Japan, Ltd., Tokyo, Japan). 


\section{Results}

\section{Patient characteristics}

The pre-change group and post-change group comprised 83 patients and 130 patients, respectively (Table 1). There were 43 patients with ARDS (mild: 5, moderate: 11 , severe: 27$)$. We found no significant inter-group differences in patient characteristics, with the exception of SOFA score $(P=0.03)$. Table 2 shows the characteristics of the propensity score-matched patients (71 patients in each group). There were no significant differences in patient characteristics between the groups. Overall, there were 157 survivors and 56 non-survivors at ICU discharge (Table 3). The univariate analysis showed significant differences in SOFA score, ARDS severity, and source of sepsis between the survivors and non-survivors. The non-survivors included a higher prevalence of severe ARDS $(P=0.03)$ and lung infections $(P=0.04)$ than the survivors. 
Table 1

Patient characteristics in the pre-change and post-change groups $(n=213)$

\begin{tabular}{|c|c|c|c|}
\hline Variables & $\begin{array}{l}\text { Pre-change group } \\
(n=83)\end{array}$ & $\begin{array}{l}\text { Post-change group } \\
(n=130)\end{array}$ & $P$ value \\
\hline Age (years) & $67.7 \pm 12.9$ & $68.9 \pm 13.4$ & 0.52 \\
\hline Male (\%) & 69.9 & 63.1 & 0.31 \\
\hline Height (cm) & $160.6 \pm 8.2$ & $160.0 \pm 8.9$ & 0.61 \\
\hline Body weight $(\mathrm{kg})$ & $58.3 \pm 15.5$ & $55.1 \pm 11.6$ & 0.09 \\
\hline $\mathrm{BMI}\left(\mathrm{kg} / \mathrm{m}^{2}\right)$ & $22.6 \pm 5.4$ & $21.5 \pm 3.9$ & 0.10 \\
\hline SOFA score (IQR) & $8.0(6.0-10.3)$ & $9.0(7.0-12.0)$ & 0.03 \\
\hline SOFA respiratory score & $2.0(1.0-3.0)$ & $2.0(1.0-3.0)$ & 0.66 \\
\hline SOFA cardiovascular score & $1.0(0-3.0)$ & $3.0(0.5-3.0)$ & 0.001 \\
\hline SOFA liver score & $0(0-1.0)$ & $0(0-1.0)$ & 0.40 \\
\hline SOFA renal score & $0(0-2.0)$ & $1.0(0-2.0)$ & 0.60 \\
\hline SOFA coagulation score & $2.0(1.0-3.0)$ & $1.0(0-2.0)$ & 0.72 \\
\hline SOFA neurologic score & $3.0(1.0-4.0)$ & $2.0(1.0-4.0)$ & 0.82 \\
\hline \multicolumn{4}{|l|}{ ARDS severity (\%) } \\
\hline Mild & 3.6 & 1.5 & \multirow[t]{3}{*}{0.16} \\
\hline Moderate & 4.8 & 5.4 & \\
\hline Severe & 9.6 & 14.6 & \\
\hline \multicolumn{4}{|l|}{ Source of sepsis (\%) } \\
\hline Lung & 48.2 & 37.7 & \multirow[t]{4}{*}{0.40} \\
\hline Abdomen & 33.7 & 41.5 & \\
\hline Urinary tract & 7.2 & 4.6 & \\
\hline Soft tissue & 3.6 & 10.0 & \\
\hline
\end{tabular}

Values are presented as mean \pm standard deviation for continuous variables and percentage or score (IQR) for categorical variables.

Abbreviations:

BMI, body mass index; SOFA, Sequential Organ Failure Assessment; ARDS, acute respiratory distress syndrome; IQR, interquartile range. 


\begin{tabular}{|lll|}
\hline Variables & $\begin{array}{l}\text { Pre-change group } \\
(\mathbf{n}=\mathbf{8 3})\end{array}$ & $\begin{array}{l}\text { Post-change group } \\
(\mathbf{n = 1 3 0 )}\end{array}$ \\
\hline Mediastinum & 1.2 & 0 \\
\hline Endocardium & 1.2 & 3.1 \\
\hline Others & 4.8 & 3.1 \\
\hline $\begin{array}{l}\text { Values are presented as mean } \pm \text { standard deviation for continuous variables and percentage or score } \\
\text { (IQR) for categorical variables. }\end{array}$ & \\
\hline Abbreviations: & \\
\hline $\begin{array}{l}\text { BMI, body mass index; SOFA, Sequential Organ Failure Assessment; ARDS, acute respiratory distress } \\
\text { syndrome; IQR, interquartile range. }\end{array}$ \\
\hline
\end{tabular}


Table 2

Patient characteristics in the pre-change and post-change groups after propensity score matching $(\mathrm{n}=$ 142)

\begin{tabular}{|c|c|c|c|}
\hline Variables & $\begin{array}{l}\text { Pre-change group } \\
(n=71)\end{array}$ & $\begin{array}{l}\text { Post-change group } \\
(n=71)\end{array}$ & $P$ value \\
\hline Age (years) & $67.8 \pm 12.7$ & $67.1 \pm 13.4$ & 0.76 \\
\hline Male (\%) & 69.0 & 64.8 & 0.59 \\
\hline Height (cm) & $160.6 \pm 8.5$ & $160.6 \pm 8.8$ & 0.95 \\
\hline Body weight (kg) & $58.7 \pm 16.1$ & $55.9 \pm 12.1$ & 0.25 \\
\hline $\mathrm{BMI}\left(\mathrm{kg} / \mathrm{m}^{2}\right)$ & $22.7 \pm 5.5$ & $21.6 \pm 4.2$ & 0.19 \\
\hline SOFA score (IQR) & $8.0(6.0-10.0)$ & $8.0(6.0-10.0)$ & 0.75 \\
\hline SOFA respiratory score & $2.0(1.0-3.0)$ & $2.0(1.0-3.0)$ & 0.91 \\
\hline SOFA cardiovascular score & $1.0(0-3.0)$ & $1.0(0-3.0)$ & 0.79 \\
\hline SOFA liver score & $0(0-1.0)$ & $0(0-1.0)$ & 0.41 \\
\hline SOFA renal score & $0(0-2.0)$ & $1.0(0-2.0)$ & 0.85 \\
\hline SOFA coagulation score & $1.0(0-2.0)$ & $1.0(0-2.0)$ & 0.97 \\
\hline SOFA neurologic score & $3.0(1.0-4.0)$ & $2.0(1.0-3.0)$ & 0.55 \\
\hline \multicolumn{4}{|l|}{ ARDS severity (\%) } \\
\hline Mild & 4.2 & 1.4 & \multirow[t]{3}{*}{0.36} \\
\hline Moderate & 2.8 & 7.0 & \\
\hline Severe & 11.3 & 15.5 & \\
\hline \multicolumn{4}{|l|}{ Source of sepsis (\%) } \\
\hline Lung & 49.3 & 36.6 & \multirow[t]{5}{*}{0.35} \\
\hline Abdomen & 29.6 & 39.4 & \\
\hline Urinary tract & 8.5 & 8.5 & \\
\hline Soft tissue & 4.2 & 11.3 & \\
\hline Mediastinum & 1.4 & 0 & \\
\hline \multicolumn{4}{|c|}{$\begin{array}{l}\text { Values are presented as mean } \pm \text { standard deviation for continuous variables and percentage or score } \\
\text { (IQR) for categorical variables. }\end{array}$} \\
\hline \multicolumn{4}{|c|}{$\begin{array}{l}\text { Abbreviations: BMI, body mass index; SOFA, Sequential Organ Failure Assessment; ARDS, acute } \\
\text { respiratory distress syndrome; IQR, interquartile range. }\end{array}$} \\
\hline
\end{tabular}




\begin{tabular}{|lll|}
\hline Variables & $\begin{array}{l}\text { Pre-change group } \\
(\mathbf{n = 7 1 )}\end{array}$ & $\begin{array}{l}\text { Post-change group } \\
(\mathbf{n = 7 1 )}\end{array}$ \\
\hline Endocardium & 1.4 & 1.4 \\
\hline Others & 5.6 & 2.8 \\
\hline $\begin{array}{l}\text { Values are presented as mean } \pm \text { standard deviation for continuous variables and percentage or score } \\
\text { (IQR) for categorical variables. }\end{array}$ & \\
\hline $\begin{array}{l}\text { Abbreviations: BMI, body mass index; SOFA, Sequential } \\
\text { respiratory distress syndrome; IQR, interquartile range. }\end{array}$ & Failure Assessment; ARDS, acute \\
\hline
\end{tabular}


Table 3

Patient characteristics in survivors and non-survivors at ICU discharge $(n=213)$

\begin{tabular}{|c|c|c|c|}
\hline Variables & $\begin{array}{l}\text { Survivors } \\
(n=157)\end{array}$ & $\begin{array}{l}\text { Non-survivors } \\
(n=56)\end{array}$ & $P$ value \\
\hline Age (years) & $69.1 \pm 13.5$ & $66.3 \pm 12.1$ & 0.17 \\
\hline Male (\%) & 66.2 & 64.3 & 0.79 \\
\hline Height (cm) & $160.0 \pm 8.4$ & $161.0 \pm 9.0$ & 0.45 \\
\hline Body weight (kg) & $56.0 \pm 12.2$ & $57.3 \pm 16.0$ & 0.55 \\
\hline BMI $\left(\mathrm{kg} / \mathrm{m}^{2}\right)$ & $21.9 \pm 4.4$ & $21.9 \pm 4.9$ & 0.95 \\
\hline SOFA score (IQR) & $8.0(6.0-11.0)$ & $10.0(7.0-12.0)$ & 0.02 \\
\hline SOFA respiratory score & $2.0(1.0-3.0)$ & $2.0(1.0-3.0)$ & 0.05 \\
\hline SOFA cardiovascular score & $2.0(0-3.0)$ & $3.0(0-3.0)$ & 0.44 \\
\hline SOFA liver score & $0(0-1.0)$ & $0(0-1.0)$ & 0.27 \\
\hline SOFA renal score & $0(0-2.0)$ & $1.0(0-2.0)$ & 0.11 \\
\hline SOFA coagulation score & $1.0(0-2.0)$ & $1.0(0-3.0)$ & 0.04 \\
\hline SOFA neurologic score & $2.0(1.0-4.0)$ & $2.0(1.0-4.0)$ & 0.61 \\
\hline \multicolumn{4}{|l|}{ ARDS severity (\%) } \\
\hline Mild & 2.5 & 1.8 & \multirow[t]{3}{*}{0.03} \\
\hline Moderate & 5.7 & 3.6 & \\
\hline Severe & 7.6 & 26.8 & \\
\hline \multicolumn{4}{|l|}{ Source of sepsis (\%) } \\
\hline Lung & 38.2 & 51.8 & \multirow[t]{6}{*}{0.04} \\
\hline Abdomen & 41.4 & 30.4 & \\
\hline Urinary tract & 6.4 & 3.6 & \\
\hline Soft tissue & 9.6 & 1.8 & \\
\hline Mediastinum & 0.6 & 0 & \\
\hline Endocardium & 1.9 & 3.6 & \\
\hline \multicolumn{4}{|c|}{$\begin{array}{l}\text { Values are presented as mean } \pm \text { standard deviation for continuous variables and number (percentag } \\
\text { or score (IQR) for categorical variables. }\end{array}$} \\
\hline
\end{tabular}




\begin{tabular}{|lll|}
\hline Variables & $\begin{array}{l}\text { Survivors } \\
(\mathbf{n = 1 5 7 )}\end{array}$ & $\begin{array}{l}\text { Non-survivors } \\
(\mathbf{n = 5 6})\end{array}$ \\
\hline Others & 1.9 & 8.9 \\
\hline $\begin{array}{l}\text { Values are presented as mean } \pm \text { standard deviation for continuous variables and number (percentage) } \\
\text { or score (IQR) for categorical variables. }\end{array}$ & \\
\hline $\begin{array}{l}\text { Abbreviations: ICU, intensive care unit; BMI, body mass index; SOFA, Sequential Organ Failure } \\
\text { Assessment; ARDS, acute respiratory distress syndrome; IQR, interquartile range. }\end{array}$ \\
\hline
\end{tabular}

\section{Mechanical ventilation measurements}

Table 4 shows the PIP levels, plateau pressure levels, and P/F ratios in the pre-change and post-change groups. None of the outcome measures exhibited a normal distribution with the Shapiro-Wilk test.

Therefore, the Mann-Whitney test was used to compare these outcomes. The post-change group, when compared with the pre-change group, showed a significant reduction in PIP just after tracheal intubation (20.0 [IQR: $20.0-25.0] \mathrm{cmH}_{2} \mathrm{O}$ vs. 25.0 [IQR: $\left.20.0-27.0\right] \mathrm{cmH}_{2} \mathrm{O}, P<0.001$ ) and maximum PIP within 48 hours (23.0 [IQR: 20.0-25.0] $\mathrm{cmH}_{2} \mathrm{O}$ vs. 25.0 [IQR: 25.0-30.0] $\mathrm{cmH}_{2} \mathrm{O}, P<0.001$ ). The P/F ratio just before tracheal intubation was higher in the post-change group than the pre-change group (183.3 [IQR: 91.0$369.4]$ vs. 154.0 [IQR: 89.2-279.9], $P=0.19$ ). There were no significant differences in the $\mathrm{P} / \mathrm{F}$ ratio just after tracheal intubation $(P=0.58)$ and at maximum PIP within 48 hours $(P=0.38)$. Furthermore, there was no significant inter-group difference in barotrauma incidence $(P=0.91)$. 
Table 4

Patient outcomes in the pre-change and post-change groups $(n=213)$

\begin{tabular}{|c|c|c|c|}
\hline Variables & $\begin{array}{l}\text { Pre-change group } \\
(n=83)\end{array}$ & $\begin{array}{l}\text { Post-change group } \\
(n=130)\end{array}$ & $P$ value \\
\hline \multicolumn{4}{|l|}{ PIP (IQR) $\left(\mathrm{cmH}_{2} \mathrm{O}\right)$} \\
\hline Just after tracheal intubation & $25.0(20.0-27.0)$ & $20.0(20.0-25.0)$ & $<0.001$ \\
\hline Max PIP within 48 hours & $25.0(25.0-30.0)$ & $23.0(20.0-25.0)$ & $<0.001$ \\
\hline \multicolumn{4}{|l|}{ Plateau pressure (IQR) $\left(\mathrm{cmH}_{2} \mathrm{O}\right)$} \\
\hline Just after tracheal intubation & $23.0(14.0-24.0)$ & $16.0(11.0-23.0)$ & $<0.001$ \\
\hline At max PIP within 48 hours & $23.0(23.0-28.0)$ & $19.0(13.0-24.0)$ & $<0.001$ \\
\hline \multicolumn{4}{|l|}{$\mathrm{P} / \mathrm{F}$ ratio (IQR) } \\
\hline Just before tracheal intubation & $154.0(89.2-279.9)$ & $183.3(91.0-369.4)$ & 0.19 \\
\hline Just after tracheal intubation & $227.0(153.0-313.9)$ & $237.0(149.5-349.2)$ & 0.58 \\
\hline At max PIP within 48 hours & $250.0(158.3-385.4)$ & $243.3(148.4-340.0)$ & 0.38 \\
\hline Barotrauma (\%) & 12.0 & 11.5 & 0.91 \\
\hline Duration of MV (IQR) (days) & $11.0(6.0-19.0)$ & $7.0(3.0-14.0)$ & 0.01 \\
\hline ICU stay (IQR) (days) & $11.0(6.0-19.0)$ & $9.0(4.0-15.0)$ & 0.02 \\
\hline Hospital stay (IQR) (days) & $40.0(24.0-69.0)$ & $44.0(22.0-73.0)$ & 0.77 \\
\hline ICU mortality (\%) & 31.3 & 23.1 & 0.18 \\
\hline In-hospital mortality (\%) & 44.6 & 44.6 & 1.00 \\
\hline \multicolumn{4}{|c|}{$\begin{array}{l}\text { Values are presented as median (IQR) for continuous variables and percentage for categorical } \\
\text { variables. }\end{array}$} \\
\hline
\end{tabular}

After propensity score matching, the post-change group, when compared with the pre-change group, showed a significant reduction in PIP just after tracheal intubation (20.0 [IQR: 18.0-25.0] $\mathrm{cmH}_{2} \mathrm{O}$ vs. 25.0 [IQR: 20.0-26.0] $\left.\mathrm{cmH}_{2} \mathrm{O}, P<0.001\right)$ and maximum PIP within 48 hours $\left(22.0\right.$ [IQR:20.0-25.0] $\mathrm{cmH}_{2} \mathrm{O}$ vs. 25.0 [IQR:24.0-30.0] $\left.\mathrm{cmH}_{2} \mathrm{O}, P<0.001\right)$. There were no significant differences in the $\mathrm{P} / \mathrm{F}$ ratio just before tracheal intubation $(P=0.24)$, the $\mathrm{P} / \mathrm{F}$ ratio just after tracheal intubation $(P=0.84)$, and at maximum $\mathrm{PIP}$ within 48 hours $(P=0.27)$.

\section{ICU mortality and in-hospital mortality}


There was no statistically significant difference in ICU mortality between the pre-change and post-change groups $(23.1 \%$ vs. $31.3 \%, P=0.18)$ (Table 4$)$. In addition, the in-hospital mortality rate was identical in both groups (both $44.6 \%, P=1.00$ ).

The association between the policy change and ICU mortality was analyzed using a multivariable logistic regression model that adjusted for SOFA score and P/F ratio just before tracheal intubation. As shown in Table 5, the post-change group was not significantly associated with reductions in unadjusted ICU mortality (OR: $0.66 ; 95 \% \mathrm{Cl}: 0.36-1.22 ; P=0.18$ ) or adjusted ICU mortality (OR: $0.65 ; 95 \% \mathrm{Cl}: 0.33-1.31 ; P$ $=0.23$ ). A similar lack of association was observed in the propensity score-matched analysis (Table 6) and subgroup analysis of ARDS patients (Table 7). The Kaplan-Meier curves of ICU mortality for the prechange and post-change groups are shown in Fig. 1. The post-change group did not have significantly better survival than the pre-change group (log-rank test: $P=0.92$ ).

Table 5

Results of the logistic regression analysis of ICU mortality $(n=213)$

\begin{tabular}{|llll|}
\hline & Odds ratio & $95 \% \mathrm{Cl}$ & Pvalue \\
\hline Unadjusted & & & \\
\hline Post-change group (ref. Pre-change group) & 0.70 & $0.22-2.23$ & 0.55 \\
\hline Adjusted & & & \\
\hline Post-change group (ref. Pre-change group) & 0.67 & $0.33-1.43$ & 0.31 \\
\hline Age & 0.98 & $0.95-1.00$ & 0.09 \\
\hline Male (ref. Female) & 1.04 & $0.46-2.35$ & 0.93 \\
\hline BMI & 1.01 & $0.93-1.09$ & 0.81 \\
\hline Source of sepsis (ref. Abdomen) & & & \\
\hline Lung & 1.04 & $0.42-2.56$ & 0.93 \\
\hline Urinary tract & 0.64 & $0.12-3.46$ & 0.61 \\
\hline Soft tissue & 0.33 & $0.04-2.98$ & 0.32 \\
\hline Mediastinum & - & - & - \\
\hline Endocardium & 2.75 & $0.36-21.0$ & 0.33 \\
\hline Others & 8.72 & $1.15-66.2$ & 0.04 \\
\hline SOFA score & 1.15 & $1.02-1.29$ & 0.02 \\
\hline P/F just before tracheal intubation & 0.99 & $0.99-1.00$ & 0.04 \\
\hline Abbreviations: ICU, intensive care unit; Cl, confidence interval; BMl, body & mass index; SOFA, Sequential \\
\hline Organ Failure Assessment; P/F, partial pressure of arterial oxygen/fraction of inspiratory oxygen.
\end{tabular}


Table 6

Results of the logistic regression analysis of ICU mortality in propensity score-matched patients $(n=$ 142)

\begin{tabular}{|llll|}
\hline & Odds ratio & $95 \%$ Cl & Pvalue \\
\hline Unadjusted & & & \\
\hline Post-change group (ref. Pre-change group) & 0.73 & $0.34-1.59$ & 0.43 \\
\hline Adjusted & & & \\
\hline Post-change group (ref. Pre-change group) & 0.81 & $0.32-2.04$ & 0.66 \\
\hline Age & 0.96 & $0.92-0.99$ & 0.02 \\
\hline Male (ref. Female) & 1.02 & $0.34-3.05$ & 0.98 \\
\hline BMI & 1.02 & $0.93-1.12$ & 0.67 \\
\hline Source of sepsis (ref. Abdomen) & & & \\
\hline Lung & 2.73 & $0.70-10.67$ & 0.15 \\
\hline Urinary tract & 1.38 & $0.20-9.39$ & 0.74 \\
\hline Soft tissue & 1.19 & $0.10-14.51$ & 0.89 \\
\hline Mediastinum & - & - & - \\
\hline Endocardium & 5.78 & $0.24-138.72$ & 0.28 \\
\hline Others & 14.61 & $1.40-152.87$ & 0.03 \\
\hline SOFA score & 1.18 & $1.01-1.38$ & 0.04 \\
\hline P/F just before tracheal intubation & 0.99 & $0.99-1.00$ & 0.47 \\
\hline $\begin{array}{l}\text { Abbreviations: ICU, intensive care unit; Cl, confidence interval; BMI, body } \\
\text { Organ Fass index; SOFA, Sequential }\end{array}$ \\
\hline
\end{tabular}


Table 7

Results of the logistic regression analysis of ICU mortality in ARDS patients $(n=50)$

\begin{tabular}{|lccc|}
\hline & Odds ratio & $95 \% \mathrm{Cl}$ & Pvalue \\
\hline Unadjusted & & & \\
\hline $\begin{array}{l}\text { Post-change group (ref. Pre-change group) } \\
\text { Adjusted }\end{array}$ & 0.66 & $0.36-1.22$ & 0.18 \\
\hline $\begin{array}{l}\text { Post-change group (ref. Pre-change group) } \\
\text { SOFA score }\end{array}$ & 0.65 & $0.33-1.31$ & 0.23 \\
\hline $\begin{array}{l}\text { P/F just before tracheal intubation } \\
\text { Abbreviations: ICU, intensive care unit; ARDS, acute respiratory distress syndrome; Cl, confidence } \\
\text { interval; SOFA, Sequential Organ Failure Assessment; P/F, partial pressure of arterial oxygen/fraction } \\
\text { of inspiratory oxygen. }\end{array}$ & $1.03-1.28$ & 0.01 \\
\hline
\end{tabular}

\section{Duration of mechanical ventilation}

As shown in Table 4, there was a significant difference in mechanical ventilation duration between the pre-change and post-change groups (11.0 [IQR: $6.0-19.0$ ] days vs. 7.0 [IQR: $3.0-14.0]$ days, $P=0.01$ ). The median mechanical ventilation duration among the survivors at ICU discharge was less than 10 days (Table 8). The Kaplan-Meier curves of time to weaning from mechanical ventilation are shown in Fig. 2. Patients in the post-change group had a significantly shorter time to weaning from mechanical ventilation than those in the pre-change group (log-rank test: $P=0.02$ ). When stratified according to the $\mathrm{P} / \mathrm{F}$ ratios just before tracheal intubation (Figs. 3, 4, and 5), we detected a significant inter-group difference in time to weaning from mechanical ventilation among patients with $P / F$ ratios of 100.1-200 (Fig. 4); however, there were no significant differences in patients with P/F ratios $>200$ and $\leq 100$ (Figs. 3 and 5). 
Table 8

Patient outcomes in survivors and non-survivors at ICU discharge $(n=213)$

\begin{tabular}{|llll|}
\hline Variables & $\begin{array}{l}\text { Survivors } \\
(\mathbf{n}=\mathbf{1 5 7})\end{array}$ & $\begin{array}{l}\text { Non-survivors } \\
(\mathbf{n = 5 6})\end{array}$ & Pvalue \\
\hline PIP (IQR) $\left(\mathrm{cmH}_{2} \mathrm{O}\right)$ & & & 0.01 \\
\hline Just after tracheal intubation & $20.0(20.0-25.0)$ & $25.0(20.0-27.8)$ & $<0.001$ \\
\hline Max PIP within 48 hours & $25.0(20.0-26.5)$ & $25.0(25.0-30.8)$ & $<0.01$ \\
\hline P/F ratio (IQR) & & & 0.02 \\
\hline Just before tracheal intubation & $195.5(112.0-356.2)$ & $124.3(65.0-257.3)$ & $<0.01$ \\
\hline Just after tracheal intubation & $247.1(169.5-345.0)$ & $165.5(126.2-318.8)$ & 0.10 \\
\hline At max PIP within 48 hours & $272.0(183.8-372.7)$ & $174.4(120.8-313.5)$ & 0.03 \\
\hline Barotrauma (\%) & 9.6 & 17.9 & 0.20 \\
\hline Duration of MV (IQR) (days) & $7.0(3.0-14.0)$ & $11.5(4.5-17.0)$ & \\
\hline ICU stay (IQR) (days) & $9.0(5.0-15.5)$ & $12.5(3.3-17.8)$ & \\
\hline $\begin{array}{l}\text { Values are presented as mean } \pm \text { standard deviation for continuous variables and percentage for } \\
\text { categorical variables. }\end{array}$ & & & \\
\hline $\begin{array}{l}\text { Abbreviations: ICU, intensive care unit; PIP, peak inspiratory pressure; IQR, interquartile range; P/F, } \\
\text { partial pressure of arterial oxygen/fraction of inspiratory oxygen; MV, mechanical ventilation. }\end{array}$
\end{tabular}

\section{ICU stay and hospital stay}

As shown in Table 4, there was a significant difference in ICU stay between the pre-change and postchange groups (11.0 [IQR: $6.0-19.0$ ] days vs. 9.0 [IQR: $4.0-15.0$ ] days, $P=0.02$ ). There was no significant difference in ICU stay between the survivors and non-survivors (9.0 [IQR: 5.0-15.5] days vs. 12.5 [IQR: 3.3-17.8] days, $P=0.20$ ) (Table 8). Similarly, there was no significant difference in hospital stay between the pre-change and post-change groups (40.0 [IQR: 24.0-69.0] days vs. 44.0 [IQR: 22.0-73.0] days, $P=$ 0.77) (Table 4).

\section{Discussion}

In this retrospective before-and-after study, we examined the differences in ICU mortality and other outcomes between septic patients who had undergone mechanical ventilation with conventional oxygenation targets and those who had undergone mechanical ventilation with permissive hypoxia and hyperoxia avoidance. Although the latter approach did not significantly improve patient survival, it was associated with reductions in mechanical ventilation duration and ICU stay. Accordingly, permissive hypoxia and hyperoxia avoidance may help to increase patient turnover and access to ventilators. This 
optimization of resource utilization in the ICU is crucial for situations where ventilators and ICU beds are in low supply.

Current trials are underway to investigate the potential benefits of conservative ventilatory strategies. The Targeted OXygen therapY in Critical illness (TOXYC) study is a multicenter randomized controlled trial being conducted in the UK to compare the effects of $\mathrm{SpO}_{2}$ targets of $88-92 \%$ and $\geq 96 \%$ on outcomes in mechanically ventilated patients with respiratory failure ${ }^{11}$. Similarly, the Handling Oxygenation Targets in the Intensive Care Unit (HOT-ICU) trial in Denmark is comparing the effects of $\mathrm{PaO}_{2}$ targets of $8 \mathrm{kPa}$ $(60 \mathrm{mmHg})$ and $12 \mathrm{kPa}(90 \mathrm{mmHg})$ on 90 -day mortality in adults with hypoxemic respiratory failure $\mathrm{e}^{12}$. In a previous before-and-after study of 105 adults who required mechanical ventilation for more than 48 hours at an Australian tertiary care hospital, it was found that an $\mathrm{SpO}_{2}$ target of $90-92 \%$ was associated with significant risk reductions for new non-respiratory organ failure and 28-day mortality when compared with conventional oxygen therapy ${ }^{13}$. In contrast, an Australian multicenter randomized controlled trial found no significant differences in ICU mortality or 90-day mortality between $\mathrm{SpO}_{2}$ targets of $88-92 \%$ and $\geq 96 \%$. Although some studies did not detect any significant differences in outcomes between permissive hypoxia and conventional oxygenation strategies ${ }^{14,15}$, one study reported an association between permissive hypoxia and reduced mortality ${ }^{16}$. Another study found no significant associations between conservative oxygen therapy and reductions in mortality or hospital stay in septic patients ${ }^{17}$, which corroborates our findings. A retrospective cohort study conducted in the Netherlands reported that neither $\mathrm{FiO}_{2}$ nor positive end-expiratory pressure settings were reduced in $78 \%$ of mechanically ventilated ICU patients with $\mathrm{PaO}_{2}$ exceeding $120 \mathrm{mmHg}$ for $\mathrm{FiO}_{2}$ targets of $0.21-0.4^{18}$. We posit that permissive hypoxia and hyperoxia avoidance may contribute to the reduction of mechanical ventilation duration and ICU stay due to the continuous and aggressive management in response to each patient's oxygenation levels.

The use of high-concentration oxygen therapy is associated with pulmonary cellular damage and decreased mucus clearance, which can depress the immune system and elevate the risk of pneumonia ${ }^{19}$. In particular, severe lung injury occurs more easily for $\mathrm{PaO}_{2}$ of $450 \mathrm{mmHg}$ or more and $\mathrm{FiO}_{2}$ of 0.6 or more ${ }^{19}$. Other studies have also reported that hyperoxia after nontraumatic cardiac arrest or stroke is associated with increased mortality ${ }^{20,21}$. An analysis of immunocompromised patients found that highconcentration oxygen therapy was significantly associated with increased complications, but not with mortality ${ }^{22}$. It should be noted that our hospital's policy does not dictate strict oxygenation control in which targets must be met at all times. Instead, it requires that physicians adjust the oxygen fractions and/or ventilatory mode settings after recognizing the occurrence of unsupportable hypoxia and hyperoxia. We found no signs of adverse events resulting from this policy.

As our ICU uses APRV for all patients with respiratory failure, this study is characterized by its focus on APRV-treated cases. Extracorporeal membrane oxygenation was not used in our patients during the study period. Previous studies have shown that APRV allows the continuous and rapid improvement of 
oxygenation in ARDS patients ${ }^{22-24}$. The use of APRV in our respiratory failure patients (including ARDS patients) may have contributed to improvements in oxygenation, and warrants further investigation. In addition, neuromuscular blocking drugs were only used in 3 emergent intubation cases (pre-change: 1 , post-change: 2). Prone positioning was performed for all patients in both groups by nursing staff. However, prone positioning for more than 12 hours can place a heavy workload on medical staff, and was not utilized in cases where patient safety could not be ensured. In this way, there were no prominent inter-group differences in the use of therapies that could potentially affect respiratory failure rates.

Our findings should be considered in the context of several limitations. First, this was a retrospective single-center study with a relatively small sample size, and our results may lack generalizability. Second, the before-and-after design of this study may have introduced biases such as observer bias or history bias. To reduce these potential biases and improve the validity of our findings, we employed multivariate logistic regression analyses and propensity score matching to account for variations in baseline characteristics. However, multicenter prospective studies are needed to confirm or refute our findings. Third, there may be confounding factors that were not included in analysis. For example, the analysis did not account for differences in underlying disease. Severe diseases such as chronic heart failure, obstructive and/or restrictive pulmonary disease, and chronic kidney failure would affect patient prognosis. In addition, the management of critically ill patients (e.g., early mobilization) may have improved over time, and it is possible that this and other unidentified confounding factors had influenced the mechanical ventilation duration and ICU stay during the relatively long study period. Fourth, we did not use any other ventilatory mode apart from APRV. Therefore, our results may have generalizability issues for other ventilatory modes. Finally, we could not explain why reductions in mechanical ventilation duration and ICU stay did not lead to corresponding reductions in hospital stay. In Japan, acute care hospitals also fulfill the roles that would be assumed by subacute care hospitals in other countries. Therefore, these longer hospitalization durations may have been less affected by the shorter ICU stay.

\section{Conclusions}

The policy of permissive hypoxia and hyperoxia avoidance for septic patients requiring mechanical ventilation did not significantly improve prognosis. However, the shift in oxygenation targets was associated with reductions in mechanical ventilation duration and ICU stay. This would increase access to ventilators and other resources in the ICU. Nevertheless, further studies using larger study populations and multiple institutions are needed to verify our findings.

\section{Declarations}

\section{Data availability}

The data set used in this study is available from the corresponding author on reasonable request.

\section{Acknowledgements}


We thank the patients and their families for their participation in the study.

\section{Author contributions}

$\mathrm{KN}$, Takeshi Umegaki, YN, and TK were responsible for the study concept and design. KN, Takeshi Umegaki, SO, TS, NA, Takeo Uba, TS, and MK treated the patients, participated in the collection, and collected data. KN, Takeshi Umegaki, and MK performed the statistical analysis. KN and Takeshi Umegaki wrote the manuscript. All authors undertook critical editing of the manuscript. All authors read and approved the final manuscript.

\section{Additional Information}

\section{Competing interests}

The authors declare no competing interests.

\section{References}

1. Acute Respiratory Distress Syndrome Network, Brower, R.G. et al. Ventilation with lower tidal volumes as compared with traditional tidal volumes for acute lung injury and the acute respiratory distress syndrome. N Engl J Med. 342, 1301-1308 (2000).

2. Ursin, Rein, P., Jacobsen, D., Ormaasen, V. \& Dunlop, O. Pneumococcal sepsis requiring mechanical ventilation: Cohort study in 38 patients with rapid progression to septic shock. Acta Anaesthesiol Scand. 62, 1428-1435 (2018).

3. Hernández, G. et al. Effect of resuscitation strategy targeting peripheral perfusion status vs serum lactate levels on 28-day mortality among patients with septic shock: The ANDROMEDA-SHOCK randomized clinical trial. 321, 654-664 (2019).

4. Martin, D.S. et al. Protocol for a feasibility randomised controlled trial of targeted oxygen therapy in mechanically ventilated critically ill patients. BMJ Open. 9, e021674 (2019).

5. Martin, D.S. \& Grocott, M.P. Oxygen therapy in critical illness: precise control of arterial oxygenation and permissive hypoxia. Crit Care Med. 41, 423-432 (2013).

6. Collins, J.A., Rudenski, A., Gibson, J., Howard, L. \& O’Driscoll, R. Relating oxygen partial pressure, saturation and content: the haemoglobin-oxygen dissociation curve. 11, 194-201 (2015).

7. Stolmeijer, R. et al. A systematic review of the effects of hyperoxia in acutely ill patients: Should we aim for less? Biomed Res Int. 7841295 (2018).

8. Pope, J.V. et al. Multicenter study of central venous oxygen saturation ( $\mathrm{ScvO}(2))$ as a predictor of mortality in patients with sepsis. Ann Emerg Med. 55, 40-46.el (2010).

9. Singer, M. et al. The third international consensus definitions for sepsis and septic shock (Sepsis-3). 315, 801-810 (2016). 
10. ARDS Definition Task Force, Ranieri, V.M. et al. Acute respiratory distress syndrome: the Berlin Definition. 307, 2526-2533 (2012).

11. Martin, D.S. et al. Protocol for a feasibility randomised controlled trial of targeted oxygen therapy in mechanically ventilated critically ill patients. BMJ Open. 9, e021674 (2019).

12. Schjørring, O.L. et al. The handling oxygenation targets in the intensive care unit (HOT-ICU) trial: Detailed statistical analysis plan. Acta Anaesthesiol Scand. 64, 847-856 (2020).

13. Suzuki, S. et al. Conservative oxygen therapy in mechanically ventilated patients: a pilot before-andafter trial. Crit Care Med. 42, 1414-1422 (2014).

14. Panwar, R. et al. Conservative versus liberal oxygenation targets for mechanically ventilated patients. A pilot multicenter randomized controlled trial. Am J Respir Crit Care Med. 193, 43-51 (2016).

15. Helmerhorst, H.J. et al. Effectiveness and clinical outcomes of a two-step implementation of conservative oxygenation targets in critically ill patients: A before and after trial. Crit Care Med. 44, 554-63 (2016).

16. Girardis, M. et al. Effect of conservative vs conventional oxygen therapy on mortality among patients in an intensive care unit: The oxygen-ICU randomized clinical trial. 316, 1583-1589 (2016).

17. Young, P. et al. Conservative oxygen therapy for mechanically ventilated adults with sepsis: a post hoc analysis of data from the intensive care unit randomized trial comparing two approaches to oxygen therapy (ICU-ROX). Intensive Care Med. 46, 17-26 (2020).

18. de Graaff, A.E., Dongelmans, D.A., Binnekade, J.M. \& de Jonge, E. Clinicians' response to hyperoxia in ventilated patients in a Dutch ICU depends on the level of $\mathrm{FiO}_{2}$. Intensive Care Med. 37, 46-51 (2011).

19. Kallet, R.H. \& Matthay, M.A. Hyperoxic acute lung injury. Respir Care. 58, 123-141 (2013).

20. Kilgannon, J.H. et al. Association between arterial hyperoxia following resuscitation from cardiac arrest and in-hospital mortality. 303, 2165-2171 (2010).

21. Rincon, F. et al. Association between hyperoxia and mortality after stroke: a multicenter cohort study. Crit Care Med. 42, 387-396 (2014).

22. Yehya, N., Topjian, A.A., Thomas, N.J. \& Friess, S.H. Improved oxygenation 24 hours after transition to airway pressure release ventilation or high-frequency oscillatory ventilation accurately discriminates survival in immunocompromised pediatric patients with acute respiratory distress syndrome. Pediatr Crit Care. 15, e147-156 (2014).

23. Ferdowsali, K. \& Modock, J. Airway pressure release ventilation: improving oxygenation: indications, rationale, and adverse events associated with airway pressure release ventilation in patients with acute respiratory distress syndrome for advance practice nurses. Dimens Crit Care Nurs. 32, 222-228 (2013).

24. Sydow, M., Burchardi, H., Ephraim, E., Zielmann, S. \& Crozier, T.A. Long-term effects of two different ventilatory modes on oxygenation in acute lung injury. Comparison of airway pressure release ventilation and volume-controlled inverse ratio ventilation. Am J Respir Crit Care Med. 149, 15501556 (1994). 
Figures

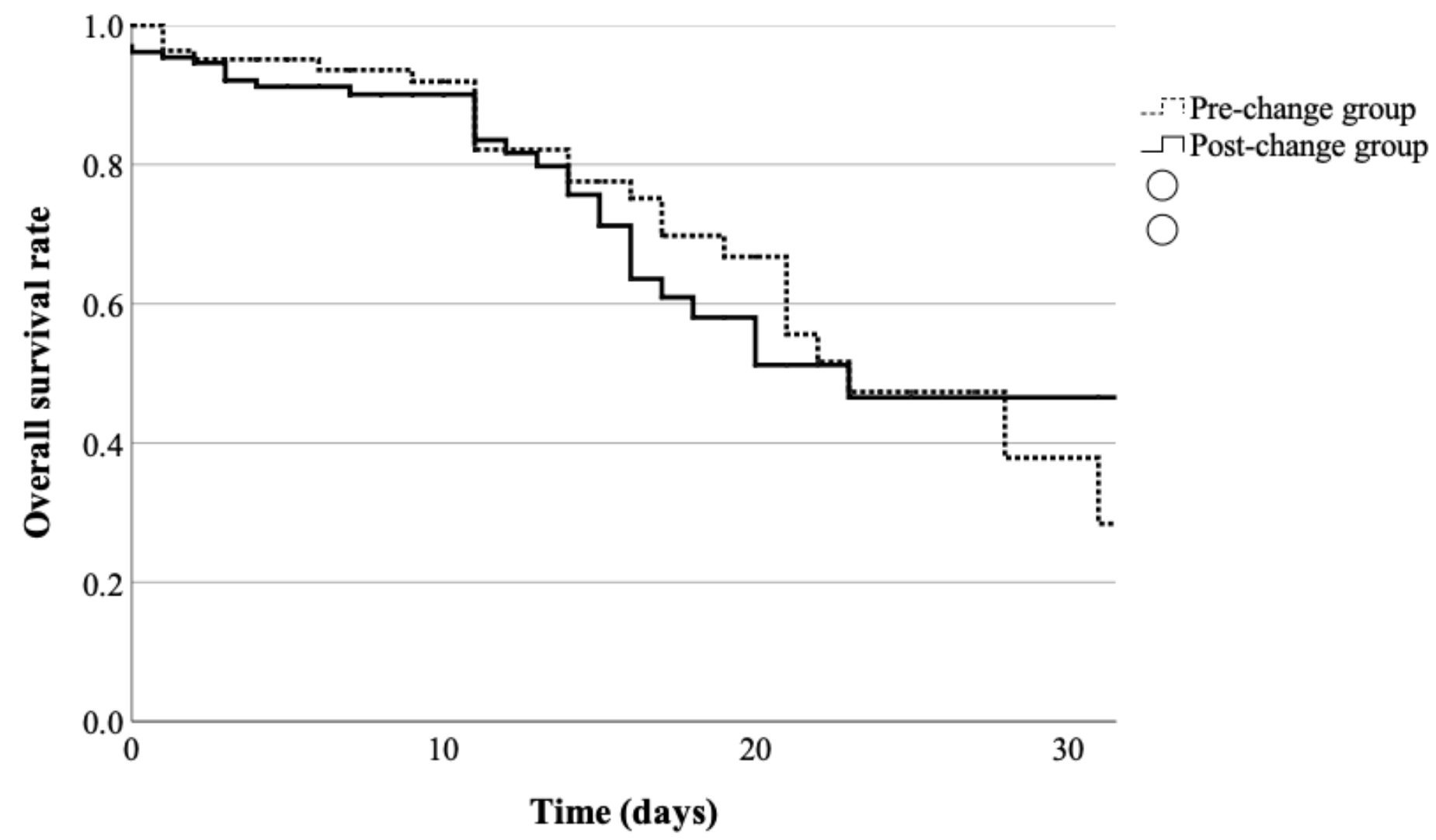

\section{Figure 1}

Kaplan-Meier ICU survival curves. There was no significant difference in survival between the pre-change and post-change groups (log-rank test: $\mathrm{P}=0.92)$. ICU, intensive care unit. 


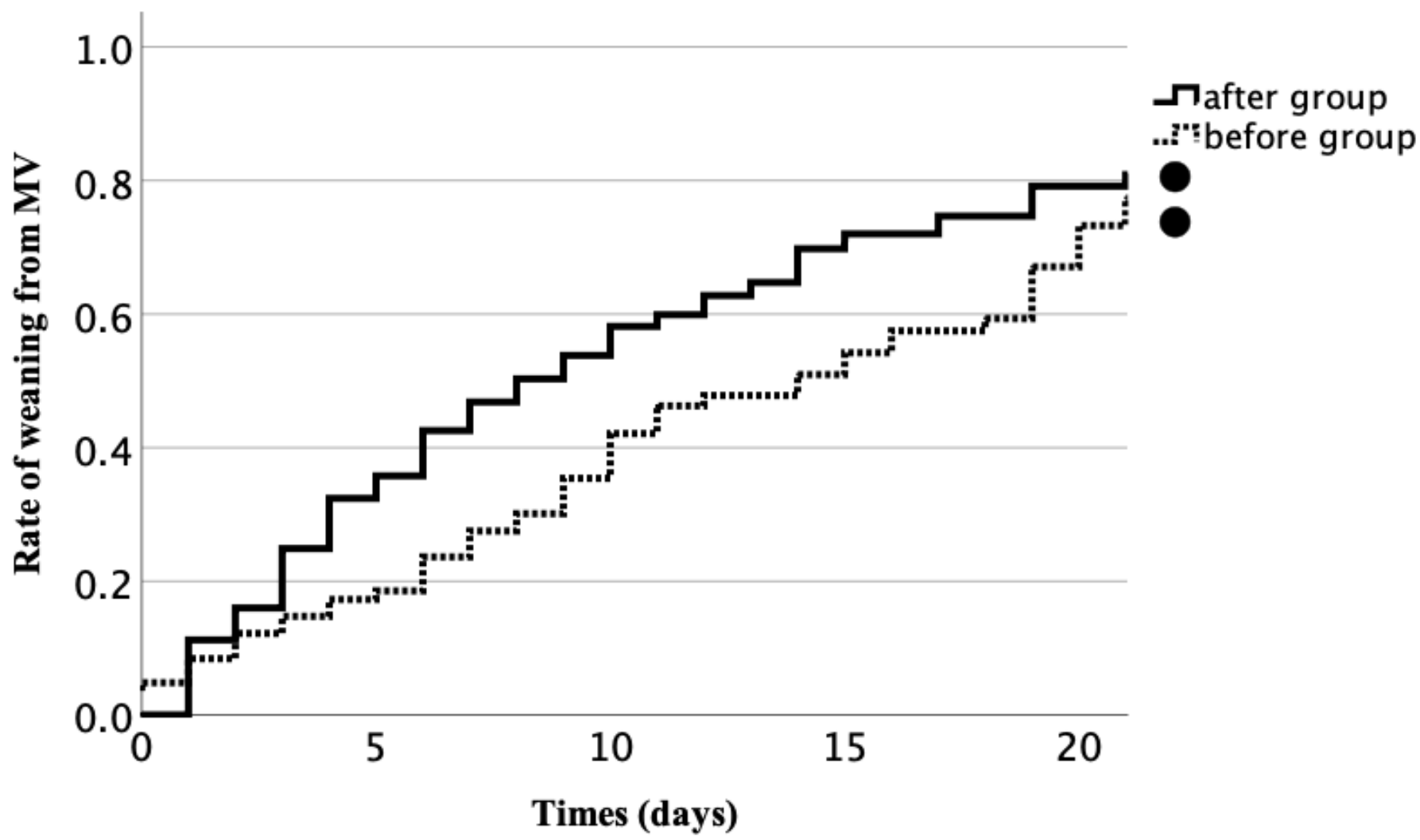

Figure 2

Kaplan-Meier curves for time to weaning from mechanical ventilation. Patients in the post-change group had significantly shorter time to weaning from mechanical ventilation than patients in the pre-change group (log-rank test: $\mathrm{P}=0.02$ ). MV, mechanical ventilation. 


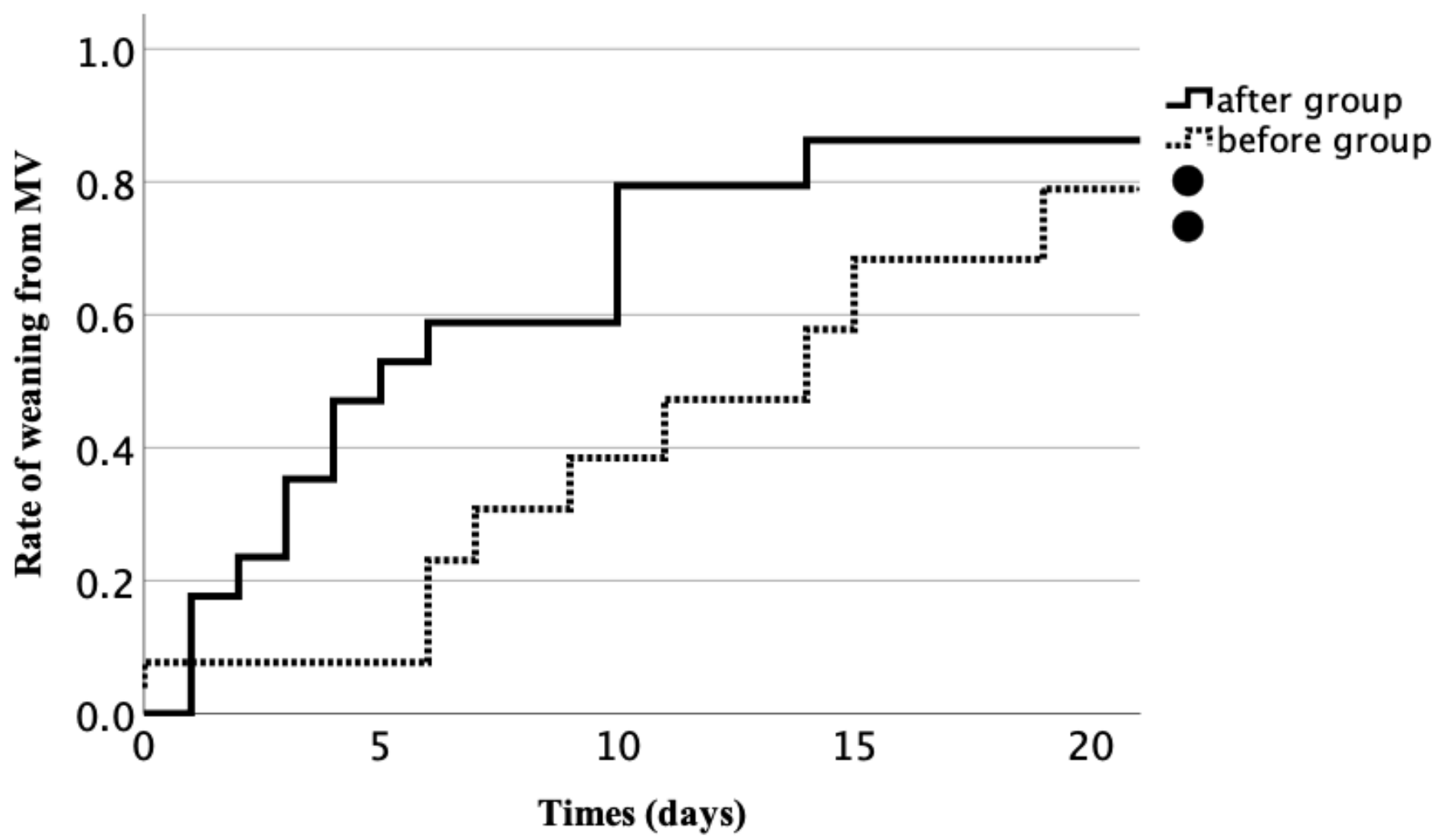

Figure 3

Kaplan-Meier curves for time to weaning from mechanical ventilation in patients with $\mathrm{P} / \mathrm{F}$ ratios $>200$. $\mathrm{P} / \mathrm{F}$ ratios were taken just before tracheal intubation. There was no significant difference in time to weaning from mechanical ventilation between the pre-change and post-change groups (log-rank test: $\mathrm{P}=0.12)$. MV, mechanical ventilation. 


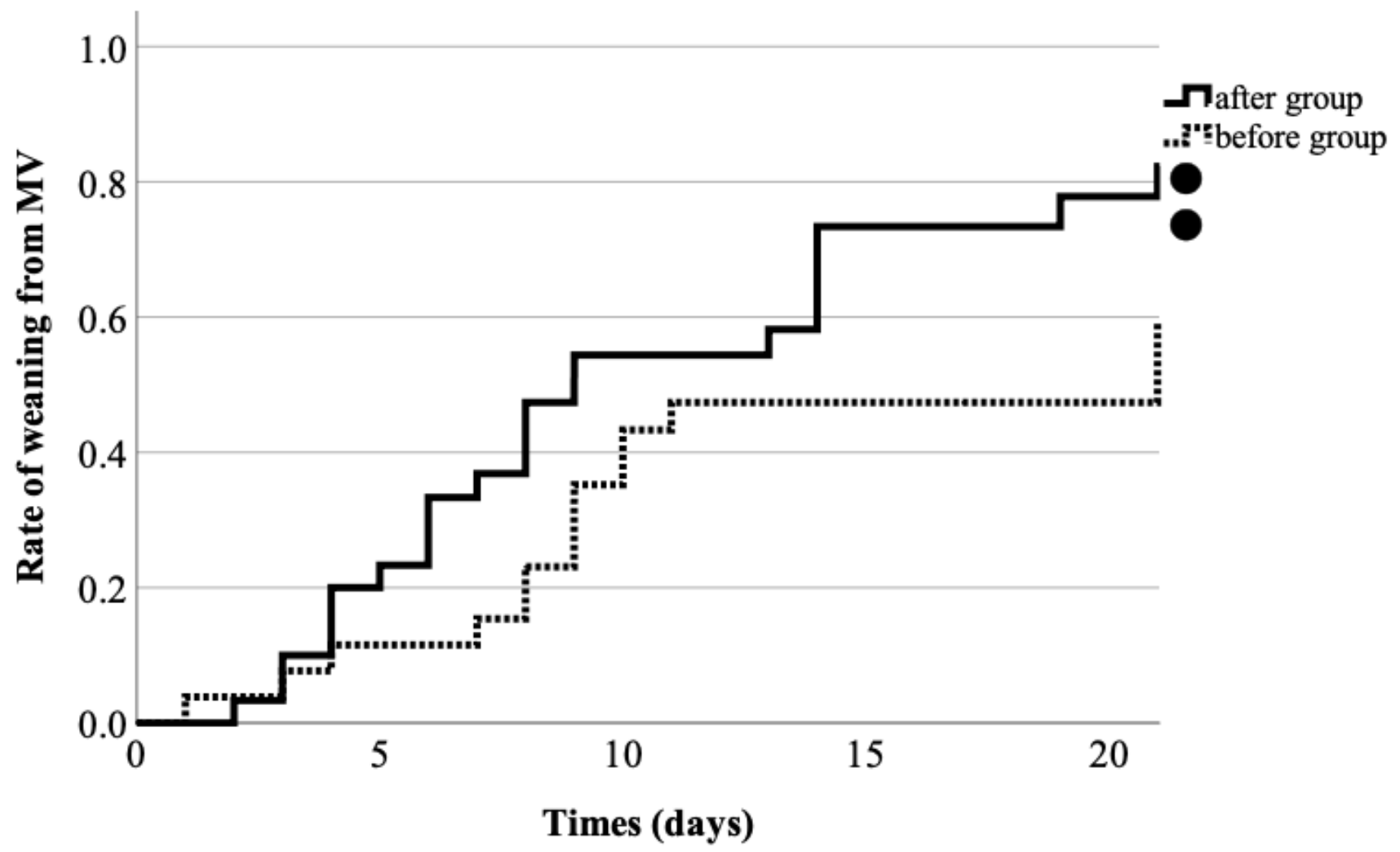

Figure 4

Kaplan-Meier curves for time to weaning from mechanical ventilation in patients with P/F ratios of 100.1-200. P/F ratios were taken just before tracheal intubation. Patients in the post-change group had significantly shorter time to weaning from mechanical ventilation than patients in the pre-change group (log-rank test: $\mathrm{P}=0.04)$. MV, mechanical ventilation. 


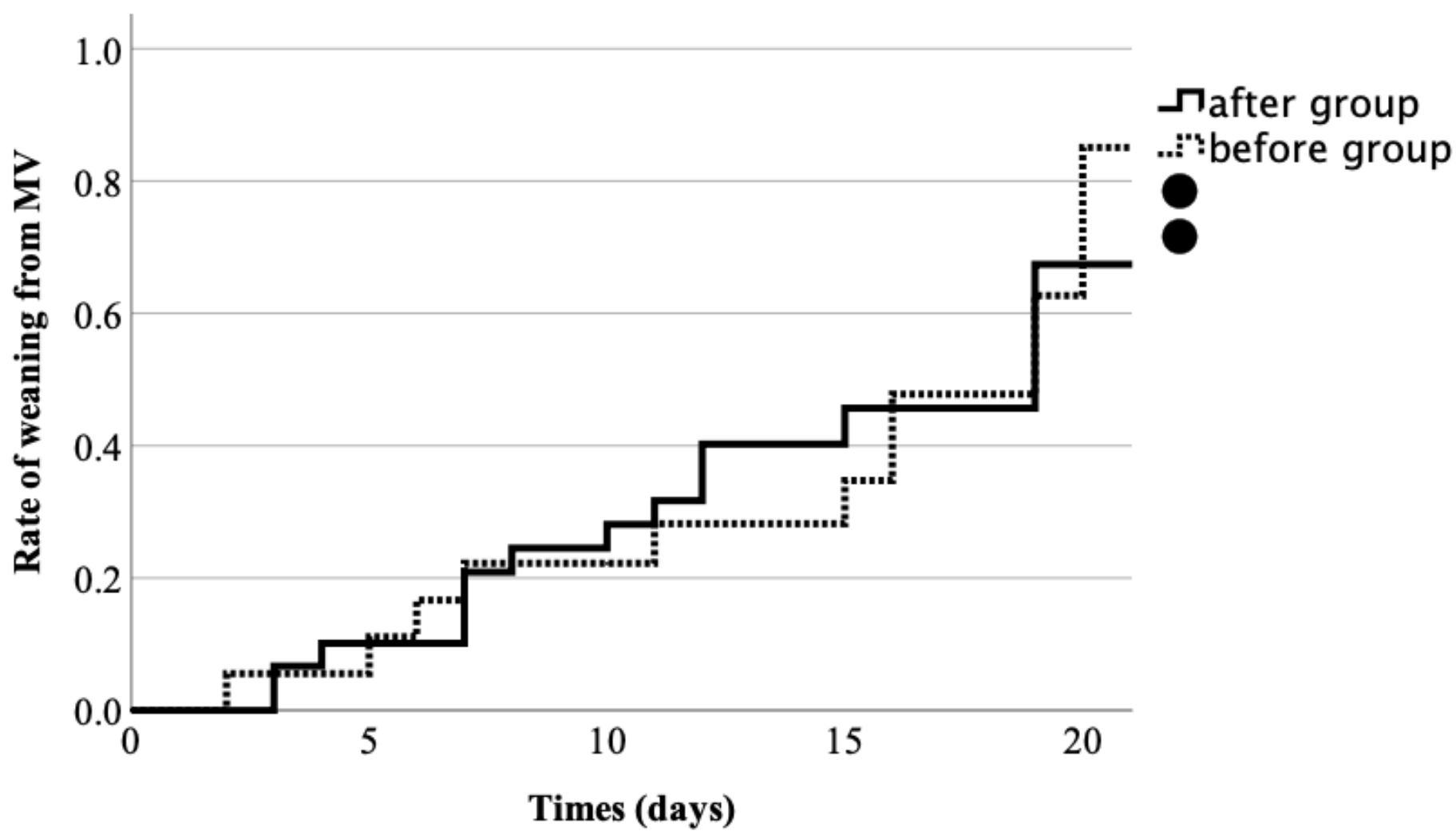

Figure 5

Kaplan-Meier curves for time to weaning from mechanical ventilation in patients with P/F ratios $\leq 100$. $\mathrm{P} / \mathrm{F}$ ratios were taken just before tracheal intubation. There was no significant difference in time to weaning from mechanical ventilation between the pre-change and post-change groups (log-rank test: $\mathrm{P}=0.98)$. MV, mechanical ventilation. 